\title{
Development of a genetic map of Vitis vinifera L. backcrossing offspring using high-throughput genotyping
}

\author{
Lytkin K.F. ${ }^{1 *}$, Grigoreva E.A. ${ }^{1,2,3}$, Potokina E.K. ${ }^{1,2}$ \\ ${ }^{1}$ Saint-Petersburg State Forestry University, St. Petersburg, Russia \\ ${ }^{2}$ All-Russian National Research Institute of Viticulture and Winemaking "Magarach" RAS, Yalta, Russia \\ ${ }^{3}$ ITMO University, St. Petersburg, Russia \\ * email: kirill.lytkin@mail.ru
}

Restriction-site associated DNA sequencing (RADseq) technology was employed to identify a large number of single nucleotide polymorphisms (SNP) for BC5 progenies of interspecies crosses between cultivated Vitis vinifera and Vitis rotundifola Michx., which is the most promising source of resistance genes in viticulture, demonstrating almost complete immunity to fungal pathogens. The set of SNP markers was further employed to construct the high-density genetic linkage maps for the $\mathrm{BC}$ crosses as a tool for further mapping of loci, inherited from the donor of resistance.

The high-density genetic linkage maps for BC5 population derived from cross of the maternal genotype 9 M. No. 31-77-10 bred at the "Magarach" Research Institute and source of resistance - paternal lines 2000-305-143 and 2000-305-163. The parental genotypes were expected to carry MrRUN1 gene (resistance to powdery mildew) and MrRPV1 gene (resistance to downy mildew), introgressed from V. rotundifola. On other hand, the parental lines may also inherit some additional loci of resistance from their male parent cv. Regent - a cultivar with quantitative resistance against downy and powdery mildew released in Germany in 1996. For cv. Regent at least two resistance loci for Erysiphe necator (Ren3, Ren 9) and one resistance locus for Plasmopara viticola (Rpv 3.1) were described.

For breeding purposes it is essential to explore genotypes of the populations obtained from crosses involving parental lines 2000-305-143 and 2000-305-163 in order to establish the position of resistance loci on chromosomes. To develop the high-density genetic linkage maps for the hybrid populations two mapping approaches were employed. First, the order of genetic markers in each of linkage group was established based on the reference $V$. vinifera $12 \mathrm{X}$ genome assembly (https://www.ncbi.nlm.nih.gov/assembly/GCF_000003745.3/). Next, the linkage map from the same set of markers was constructed using JoinMap 4 software. For the both approaches the total length of linkage maps was evaluated and compared.

Acknowledgements: The research was supported by the Russian Science Foundation (Project No. 20-16-00060). 